\title{
THE CASE OF AB AURIGAE'S DISK IN POLARIZED LIGHT: IS THERE TRULY A GAP?
}

\author{
Marshall D. Perrin ${ }^{1,2}$, Glenn Schneider ${ }^{3}$, Gaspard Duchene ${ }^{4,5}$, Christophe Pinte $^{5}$, Carol A. Grady ${ }^{6,7}$, \\ John P. WisniewsKi ${ }^{8}$, AND DeAn C. Hines $^{9}$ \\ ${ }^{1}$ Division of Astronomy, University of California, Los Angeles, CA 90095, USA; mperrin@ucla.edu \\ ${ }^{2}$ Center for Adaptive Optics, University of California, Santa Cruz, CA 95064, USA \\ ${ }^{3}$ Steward Observatory, The University of Arizona, 933 North Cherry Avenue, Tucson, AZ 85721, USA \\ ${ }^{4}$ University of California, Berkeley, Berkeley, CA 94720, USA \\ ${ }^{5}$ Université Joseph Fourier-Grenoble 1/CNRS, Laboratoire d'Astrophysique de Grenoble (LAOG) UMR 5571, BP 53, 38041 Grenoble, Cedex 09, France \\ ${ }^{6}$ Eureka Scientific, 2452 Delmer, Suite 100, Oakland, CA 96002, USA \\ ${ }^{7}$ Exoplanets and Stellar Astrophysics Laboratory, Code 667, NASA's Goddard Space Flight Center, Greenbelt, MD 20771, USA \\ ${ }^{8}$ Department of Astronomy, University of Washington Seattle, WA 98195-1580, USA \\ ${ }^{9}$ Space Science Institute, 4750 Walnut Street, Suite 205, Boulder, CO 80301, USA \\ Received 2009 October 12; accepted 2009 November 9; published 2009 December 4
}

\begin{abstract}
Using the Near-Infrared Camera and Multi-Object Spectrometer (NICMOS) coronagraph, we have obtained highcontrast $2.0 \mu \mathrm{m}$ imaging polarimetry and $1.1 \mu \mathrm{m}$ imaging of the circumstellar disk around AB Aurigae on angular scales of 0 ' $3-3^{\prime \prime}(40-550 \mathrm{AU})$. Unlike previous observations, these data resolve the disk in both total and polarized intensity, allowing accurate measurement of the spatial variation of polarization fraction across the disk. Using these observations, we investigate the apparent "gap" in the disk reported by Oppenheimer et al.. In polarized intensity, the NICMOS data closely reproduce the morphology seen by Oppenheimer et al., yet in total intensity we find no evidence for a gap in either our 1.1 or $2.0 \mu \mathrm{m}$ images. We find instead that region has lower polarization fraction, without a significant decrease in total scattered light, consistent with expectations for backscattered light on the far side of an inclined disk. Radiative transfer models demonstrate this explanation fits the observations. Geometrical scattering effects are entirely sufficient to explain the observed morphology without any need to invoke a gap or a protoplanet at that location.
\end{abstract}

Key words: circumstellar matter - planetary systems: protoplanetary disks - polarization - stars: individual (AB Aur) - stars: pre-main sequence

\section{INTRODUCTION}

$\mathrm{AB}$ Aurigae is one of the most intensively studied Herbig $\mathrm{Ae} / \mathrm{Be}$ stars, on account of its proximity, brightness, and youth (distance $d=144 \mathrm{pc}$; visual magnitude $V=7.04 \mathrm{mag}$; age $<3 \mathrm{Myr}$; spectral type A0e). Steadily improving observational capabilities have yielded increasingly detailed views of its complex and dusty environment, providing many insights into the nature of circumstellar disks (e.g., Grady et al. 1999; Fukagawa et al. 2004; Piétu et al. 2005).

In particular, Oppenheimer et al. (2008, hereafter Opp08) recently presented high angular resolution, high-contrast imaging polarimetry of $\mathrm{AB}$ Aur at $1.6 \mu \mathrm{m}$, obtained with the Lyot Project coronagraph on the Advanced Electo-Optical System (AEOS) $3.6 \mathrm{~m}$ telescope. These observations resolved the disk in polarized scattered light as close as $40 \mathrm{AU}(0.3)$ to the star. In polarized light the disk is not axisymmetric, but instead shows an apparent gap or depleted region at a radius of $\sim 100 \mathrm{AU}$. Such a gap may be created by dynamical perturbations from forming planets (e.g., Kuchner \& Holman 2003; Wyatt 2005; Jang-Condell 2009). Intriguingly, Opp08 report a faint point source within the gap, which they conjecture could be the perturbing object-though they are cautious with this identification due to its low statistical significance $(2.8 \sigma)$. The formation mechanism(s) of massive planets at large separations remain highly uncertain (Dodson-Robinson et al. 2009; Nero \& Bjorkman 2009); any prospect for observing a planet forming in situ around $\mathrm{AB}$ Aur should be pursued to clarify this puzzle.

One challenge in interpreting the data from Opp08 is that the disk is visible only in polarized intensity, $P=\sqrt{Q^{2}+U^{2}}$ (where $Q$ and $U$ are the usual Stokes parameters; see Tinbergen 1996 for a review of polarization fundamentals and notation). In their total intensity image (Stokes $I$ ), the residual speckle halo of the stellar point-spread function (PSF) completely drowns out the disk's fainter light. The origin of features seen only in polarized light is ambiguous: any observed spatial variation may be due either to variation in the total amount of scattered light, or to a change in the polarization fraction of that light. The polarization induced by dust scattering depends strongly on the scattering angle (see Figure 1), allowing disk geometry or viewing angle to cause variations in the observed polarization which might be mistaken for intrinsic substructure within the disk.

In particular, for $\mathrm{AB}$ Aur the observed celestial position angle (P.A.) of the depleted region, $333^{\circ} \pm 2^{\circ}$ (Opp08), is precisely aligned with the disk's apparent rotation axis as inferred from $\mathrm{CO}$ emission line kinematics $\left(330^{\circ}-333^{\circ}\right.$; Corder et al. 2005; Piétu et al. 2005). Is this alignment coincidental?

The most direct way to answer this question is to obtain images with enough contrast to directly detect the disk in total intensity, and then calculate the polarization fraction, $p=P / I$. Such observations are best obtained with the Hubble Space Telescope (HST), whose Advanced Camera for Surveys (ACS) and Near-Infrared Camera and Multi-Object Spectrometer (NICMOS) coronagraphs both have provided sufficiently high contrast to precisely and accurately measure the polarization of disk-scattered light (Hines et al. 2000; Schneider \& Hines 2007; Graham et al. 2007, D. C. Hines \& G. Schneider 2010 , in preparation).

In this Letter, we present new NICMOS coronagraphic imaging and polarimetry of $\mathrm{AB}$ Aur which clarifies the nature of the dark "gap" observed by Opp08. These data were obtained as part of a coronagraphic polarimetry survey of young stars across a range of masses and ages which will be reported more fully in future works (see Perrin et al. 2009, for a brief overview). 


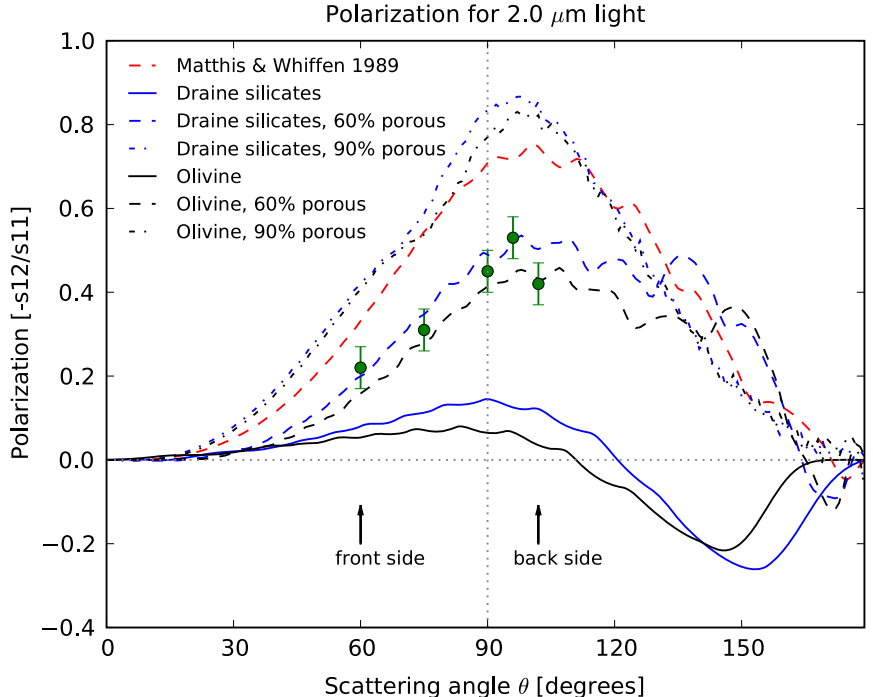

Figure 1. Induced polarization for some possible grain compositions, demonstrating that polarization depends on both scattering angle and dust grain properties, particularly composition and porosity. Positive values indicate typical centrosymmetric linear polarization, while negative formal polarization denotes linear polarization oriented radially. Polarization is maximized for porous grains, and for scattering angles slightly above $90^{\circ}$. These models assume a power-law distribution of grain sizes from 0.03 to $200 \mu \mathrm{m}$ with slope -3.5 , typical for young stellar object disks; see Section 5.2 of Pinte et al. (2008) for a discussion of the dust model and computation details. The overplotted points show the fractional polarization observed around AB Aur; the inferred scattering angles are not symmetric due to the flaring of the disk surface, estimated $\sim 10^{\circ}$. Of the models shown, the $60 \%$ porosity silicates provide the best fit.

\section{OBSERVATIONS AND DATA REDUCTION}

Our observation and reduction strategies follow the standard recommendations for NICMOS coronagraphy. We observed AB Aur on 2007 September 14 and 2007 December 21 as part of program $H S T / \mathrm{GO} 11155$, in two visits identical except for a $136^{\circ}$ difference in roll angle. After centering AB Aur behind the NIC2 coronagraph hole, nine $192 \mathrm{~s}$ exposures were taken using the $2.0 \mu \mathrm{m}$ POL $* \mathrm{~L}$ linear polarizers, cycling between the $0^{\circ}$, $120^{\circ}$, and $240^{\circ}$ polarizers after each exposure, followed by one 512 s exposure with F110W. The telescope was then shifted to move AB Aur a few arcseconds away from the occulting spot, after which we took two dithered $4 \mathrm{~s}$ exposures in both POLOL and F110W for photometry on the unocculted star.

Achieving high contrast with NICMOS requires subtracting a color-matched PSF, and for polarimetric observations, the reference star must be unpolarized to minimize systematic biases (the direct stellar light from $\mathrm{AB}$ Aur should be unpolarized or nearly so, due to the low line-of-sight extinction, $A_{V}=0.25$; Roberge et al. 2001). It proved challenging to identify PSF stars which are unpolarized yet also sufficiently red to match AB Aur's color $(H-K=0.832)$, which is redder than the RayleighJeans slope; hence anything so red must either have nonthermal emission (often highly polarized) or else be dusty and extincted (likewise polarizing). After some consideration, we identified nearby $M$ dwarfs as the best candidate PSF references, and therefore observed Proxima Centauri, GJ 273, and GJ 447 one visit each using an identical observing strategy as above. We supplemented our program with additional PSF observations drawn from programs HST/GO 10847 and 10852.

Our data reduction approach follows that of Schneider et al. (2005). Briefly, starting with pipeline-reduced images from STScI, we corrected for bad pixels and sky/thermal background emission in all images, and then median-combined the three coronagraphic images for each POL*L filter. The F110W coronagraphy and the unocculted imaging were reduced similarly.

Obtaining optimal PSF subtraction depends on accurate registration and flux-scaling. Starting from flux ratios derived from photometry of the unocculted stars, we adopted two independent strategies to optimize the subtractions: (1) a manual search visually compared subtractions of different reference PSFs while interactively varying the registration and scaling to minimize the residuals, and (2) an automated algorithm evaluated subtractions across a grid in alignment parameter space for each image pair. Each subtracted image was high-pass filtered to reject diffuse light from the disk while emphasizing features with angular scale comparable to the diffraction limit (the characteristic size of speckle residuals). The variance on this angular scale was minimized to find the best subtraction. The best subtractions were obtained using GJ 273 as the PSF; our two optimization approaches yielded subtracted data sets with polarization fractions differing by $\lesssim 6 \%$, which we adopt as our polarization uncertainty.

Remaining artifacts in the subtracted images such as diffraction spikes were masked out. The images were rectified for geometrical distortion, rotated to a common orientation, and combined to produce final mosaics in each filter. From these images the Stokes parameters $I, Q$, and $U$ were derived using the POLARIZE software (Hines et al. 2000) which models the imperfect linear polarizers in NICMOS. The output polarized images were then smoothed by a one-resolution element $(\sim 3$ pixels $=0$ '.22) Gaussian, but we present the undersampled F110W data at full resolution.

The resulting images are shown in Figure 2. The F110W subtractions are excellent, yielding a clean image with minimal PSF residuals. The POL $*$ L images are more affected by instrumental residuals due to optimization of the NIC2 coronagraphic optics for shorter wavelengths, and to a better PSF template color match at $1.1 \mu \mathrm{m}$. These factors result in $2.0 \mu \mathrm{m}$ images dappled with faint residual speckles, but that still clearly show the bright circumstellar nebulosity.

\section{RESULTS}

A complex and asymmetric nebula surrounds AB Aurigae. The F110W image clearly shows the multiple spiral arms previously observed, for instance, by Grady et al. (1999) and Fukagawa et al. (2004). The bright inner region of the disk extends out to $\sim 1^{\prime \prime} .2$, and is brightest to the south and southeast, as was seen by Fukagawa et al. At $2.0 \mu \mathrm{m}$ in total intensity (Stokes $I$ ), the overall surface brightness distribution is similar to that seen at $1 \mu \mathrm{m}$, albeit at lower angular resolution and contrast.

The $2.0 \mu \mathrm{m}$ polarized intensity image (the lower left panel of Figure 2) reproduces with high fidelity the polarized intensity pattern from Opp08. Both images show the region of lower polarized intensity at P.A. $=333^{\circ}$ between two brighter "shoulders" on either side, the brightness enhancement southwest of the star, and even the scalloped, almost-concave southern edge of the bright polarized region. Comparing the NICMOS and AEOS data sets, the NICMOS image is more sensitive and traces polarized light further from the star $\left(\sim 7^{\prime \prime}\right.$ versus $\left.\sim 1^{\prime \prime} .2\right)$, while the AEOS image has slightly better angular resolution, and better speckle rejection due to the simultaneous differential technique (Kuhn et al. 2001; Perrin et al. 2008). Yet the most significant advantage of our NICMOS observations is that they allow direct measurement of the polarization fraction as the ratio $P / I$ (Figure 2, bottom center). 


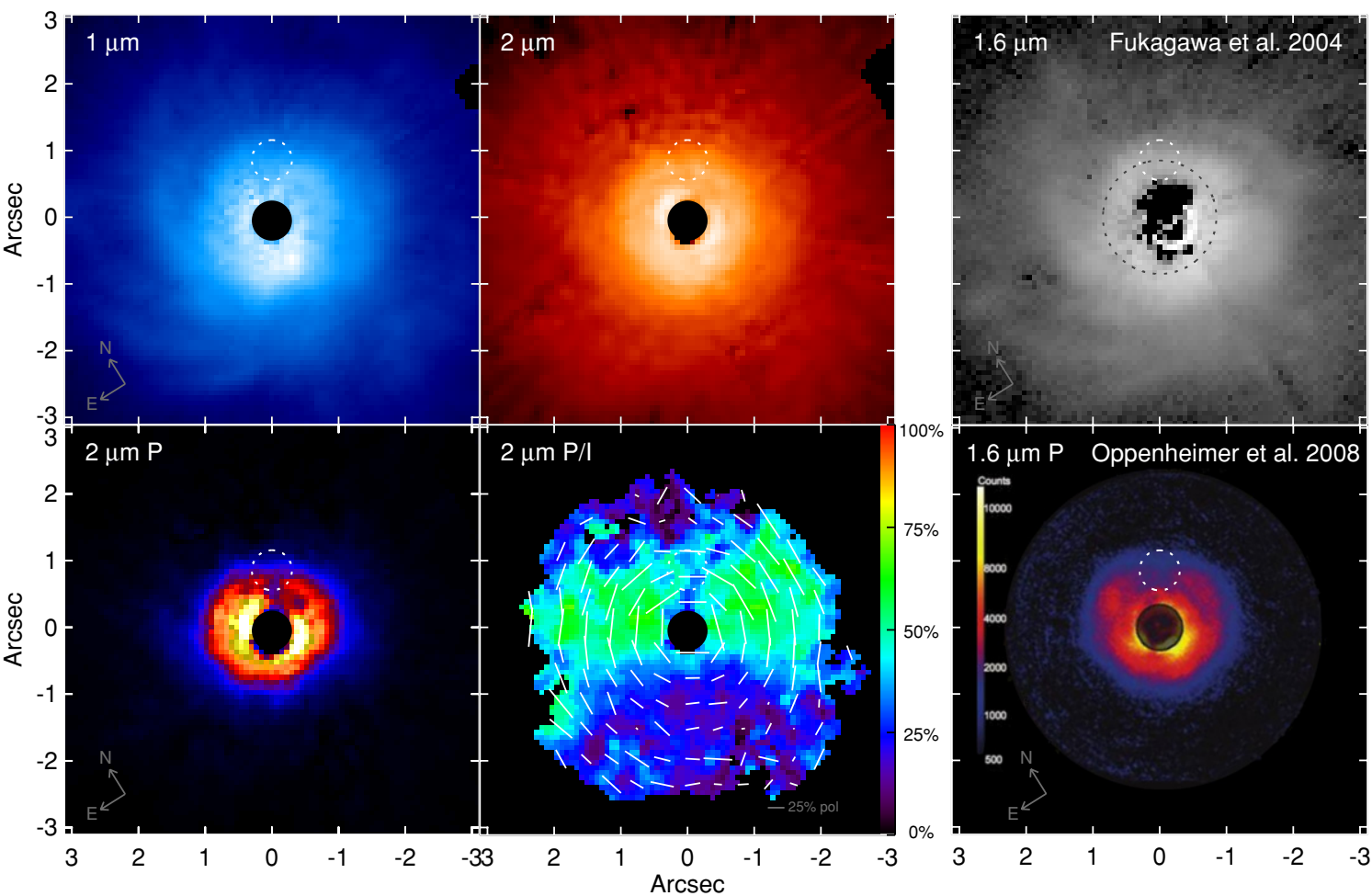

Figure 2. Near-IR imaging and polarimetry of AB Aur. Left four panels: our new NICMOS observations. These panels display the 1.1 and $2.0 \mu \mathrm{m}$ total intensities (using arcsinh stretches), $2.0 \mu \mathrm{m}$ polarized intensity (using a log stretch matching that used by Opp08), and polarization fraction (shown using a linear stretch indicated by the inset color bar and with vectors showing the polarization angles). All images have been rotated to align the disk's minor axis at P.A. $=328^{\circ}$ vertical. Right panels: $H$-band total intensity and polarized intensity from previous works, for comparison. Our $2 \mu \mathrm{m}$ polarized intensity observations reproduce the appearance seen by Opp08 very closely, but our $P / I$ image shows that the "depleted region," indicated with a white dashed circle in all panels, is in fact a region of lower polarization fraction, not lower total intensity. None of the total intensity images show any indication of an open region at that location in the disk (though we caution that in the $H$-band image from Fukagawa et al. (2004), the region of interest straddles the radius, shown in black, inside of which they state PSF subtraction artifacts rendered their data not photometrically reliable.).
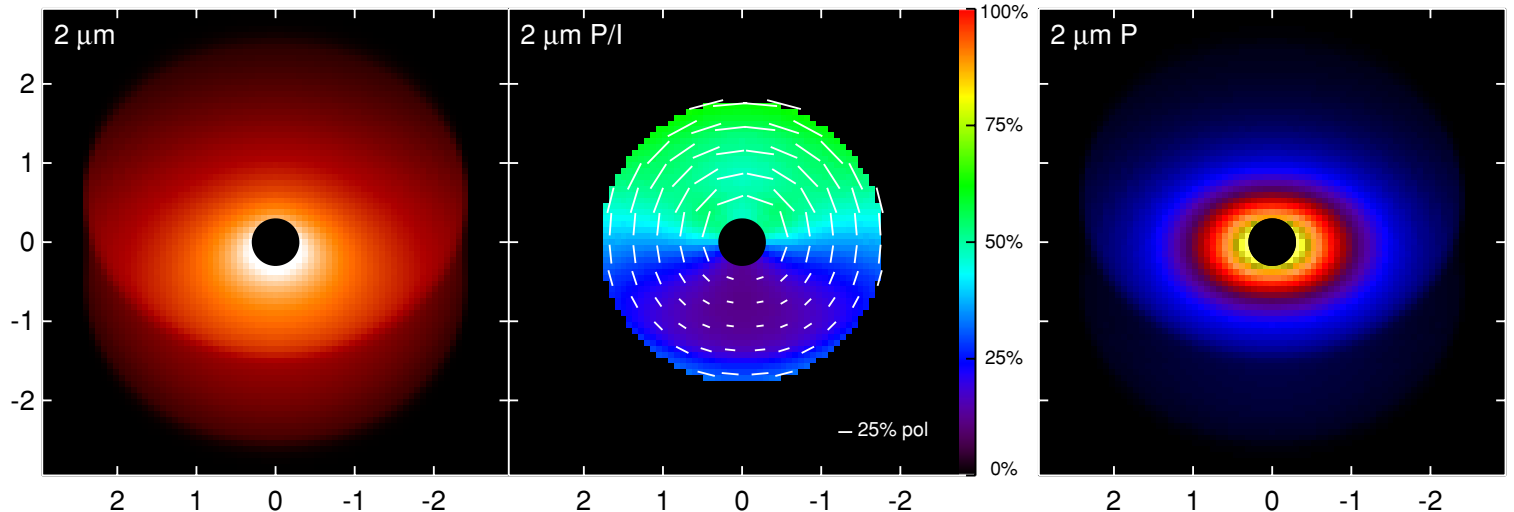

Figure 3. Our best-fit MCFOST simulated images of AB Aur, for an inclination of $35^{\circ}$ and using a population of astrosilicate grains with $60 \%$ porosity, $a_{\mathrm{max}}=1 \mu \mathrm{m}$ and $d N / d a \propto a^{-3 / 5}$. The three panels show $2 \mu \mathrm{m}$ total intensity, polarization fraction, and polarized intensity, using the same display scales as the corresponding panels in Figure 2; see Section 4.

The percentage polarization revealed this way varies strongly around $\mathrm{AB}$ Aur: The disk's southeastern half is much less polarized than the opposite side, with polarizations of $\sim 25 \% \pm$ $5 \%$ and $45 \% \pm 12 \%$, respectively. The two bright "shoulders" are seen to be regions of maximum polarization $(\sim 55 \%)$, separated by the lower polarization $(\sim 40 \%)$ region corresponding to the "depleted region" from Opp08. The symmetry axis of the overall polarization pattern is $328^{\circ} \pm 3^{\circ}$, aligned with both the "gap," and with the inferred rotation axis of the disk (Corder et al. 2005; Piétu et al. 2005). The claimed "gap" in polarized intensity is now seen to be a region of lower polarization fraction, and not a region of decreased total disk-scattered light. There is no significant decrease in surface brightness in any of the total intensity images at this location (see the white circles in Figure 2).

\section{A MODEL FOR SCATTERING FROM AB AUR}

The alignment of the polarization pattern with the disk's inclined rotational axis provides a convincing indication that scattering geometry and not disk substructure predominantly causes the observed appearance in polarized light. As shown in 
Table 1

Range of Parameters Explored in Model Grid

\begin{tabular}{ll}
\hline \hline \multicolumn{1}{c}{ Parameter } & \multicolumn{1}{c}{ Values } \\
\hline Inclination & $13^{\circ}-89^{\circ}, 20$ steps evenly spaced in $\cos i^{\circ}$ \\
Dust composition & Astrosilicates ${ }^{\mathrm{a}}$, olivine $^{\mathrm{b}}$, \\
& ISM silicates+carbon mixture $^{\mathrm{c}}$ \\
Grain porosity & $0,0.2,0.4,0.6,0.9$ \\
Grain maximum radius & $1,20,200$ \\
Grain size power law & $-2.5,-3.5,-4.5$ \\
\hline
\end{tabular}

Notes. Fixed model parameters: stellar $T_{\text {eff }}=9772 \mathrm{~K}, L_{*}=47 L_{\odot}$, dust mass $10^{-4} M_{\odot}$, disk $R_{\text {inner }}=0.2 \mathrm{AU}, R_{\text {outer }}=350 \mathrm{AU}$, scale height $h=14 \mathrm{AU}$ at $R=100 \mathrm{AU}$, disk flaring $h \propto R^{1.3}$; see Tannirkulam et al. (2008) and references therein.

a Draine \& Lee (1984) Weingartner \& Draine (2001).

b Dorschner et al. (1995).

c Mathis \& Whiffen (1989).

Figure 1, the polarization of dust-scattered light is maximized for scattering angles near or just above $90^{\circ}$, and then decreases for scattering angles closer to $180^{\circ}$. The higher polarization of the northwestern half of the disk indicates that side is tilted away from us, the most distant part of the disk, where the light is most strongly backscattered, has lower polarization without any need to invoke clearing.

To demonstrate that this scenario accounts for the observed polarization around AB Aur, we produced model images using the MCFOST Monte Carlo radiative transfer code (Pinte et al. 2006). We concentrated on fitting the $2.0 \mu \mathrm{m}$ polarization by varying dust properties, holding most disk parameters (e.g., radii, scale height, etc.) fixed based on previous modeling (e.g., Tannirkulam et al. 2008, and references therein). The inclination of $\mathrm{AB}$ Aur's disk is not precisely known: using $\mathrm{CO}$ kinematics, some authors have found inclinations as high as $33^{\circ}-40^{\circ}$ (Piétu et al. 2005; Lin et al. 2006), but others favor $21^{\circ}$ or less (Corder et al. 2005). Lower inclinations are also supported by the observed very low column density of hydrogen and high Ly $\alpha$ wind velocity (Roberge et al. 2001), and by near-infrared interferometry (Eisner et al. 2004). Due to the uncertainty, we allowed the inclination to vary as a free parameter.

We evaluated models across a grid in parameter space for plausible dust populations (see Table 1). For each model, we computed the spectral energy distribution (SED) and $2 \mu \mathrm{m}$ total and polarized images at a range of inclinations. Images were convolved with a 0 '.22 Gaussian to match the data's resolution. To find the best fit, we first discarded models which did not provide an acceptable match to AB Aur's SED, then calculated each model's azimuthal polarization profile in an $0.7-1$.'.5 annulus, and computed the $\chi^{2}$ statistic relative to the observed $2.0 \mu \mathrm{m}$ polarization profile.

The best-fit model uses silicate grains with $60 \%$ porosity, maximum grain size $1 \mu \mathrm{m}$, and a power-law size distribution with index $=-3.5$; see Figures 3 and 4 . These parameters are similar to those inferred for solar system cometary dust grains (Petrova et al. 2000; Shen et al. 2009).

We found that inclinations of $22^{\circ}-35^{\circ}$ best fit the scattered light around $\mathrm{AB}$ Aur, consistent with the range inferred from $\mathrm{CO}$ velocities. With the above dust parameters, inclinations of $\geqslant 35^{\circ}$ better reproduce the polarization drop from backscattering. Lower inclinations $\leqslant 22^{\circ}$ improve the fit to the average polarization of the near and far sides, but do not show as strong a decrease in polarization on the far side as is observed. Lower inclinations also better simultaneously fit the total intensity profile as well as polarized intensity (see Figure 4).
These slight discrepancies are most likely due to oversimplifications in our dust model: MCFOST assumes simple Mie scattering from spherical dust grains, an imperfect approximation for actual circumstellar dust, which is believed to consist of irregular fractal aggregates (Dominik et al. 2007, and references therein). Realistic scattering properties for fractal grains can be calculated through more computationally intensive techniques such as the discrete dipole model (e.g., Petrova et al. 2000; Das et al. 2008; Shen et al. 2009). Aggregate grains seem promising candidates to provide a better fit for AB Aur.

Specifically, discrete dipole calculations in some cases predict polarizations that peak at lower scattering angles $\left(90^{\circ}\right.$ or below) and decrease more strongly toward $180^{\circ}$ compared with Mie results (see, for instance, Figure 8 of Shen et al. 2009). That shift toward higher polarization at lower scattering angles would broaden the azimuthal polarization profile and could deepen a dip from backscattering, two changes that would improve our fit to AB Aur. (In Figure 4, the visible offset between the observed profile's polarization peaks, near $90^{\circ}$ and $270^{\circ}$, and the model profiles' peaks, near $135^{\circ}$ and $225^{\circ}$, directly shows the need for dust grains whose polarization maximum occurs at lower scattering angle.) Improving disk models to use more sophisticated dust treatments during radiative transfer is a logical next step.

\section{DISCUSSION}

\subsection{Structure in AB Aurigae's Disk}

The pattern of polarization resolved around AB Aur indicates unambiguously that the spatial variation of polarized light there is due primarily to the geometry of scattering from the inclined disk's surface. These data do not support the hypothesis of significant clearing in the disk near P.A. $=333^{\circ}$.

Independent of but simultaneously with this study, a numerical investigation of protoplanet shadows in disks reached a similar conclusion, finding the observed morphology around AB Aur inconsistent with the presence of any protoplanet above 0.3 Jupiter masses (H. Jang-Condell \& M. J. Kuchner 2010, in preparation).

While the previously claimed gap does not appear to be present, there is real structure within AB Aur's disk which is visible in scattered light, including polarization. Many of the asymmetric spots seen in the $2.0 \mu \mathrm{m}$ polarization fraction image coincide with the spiral arms as seen in the $1.1 \mu \mathrm{m}$ image. For instance, the northeasternmost region of high polarization, near $(1.7,0)$ in the coordinate system of Figure 2 , is precisely aligned with the brightest spiral arm. Overplotting or blinking these images shows several such alignments. Perturbations in the disk's surface, from either localized changes in scale height or warps in midplane location, could change the scattering geometry at the optical depth $\tau=1$ surface to produce the observed minor variations in polarization. For optically thick disks, surface features seen in scattered light do not necessarily correlate with conditions at the midplane (Jang-Condell \& Boss 2007), but in the case of AB Aur there is direct evidence from $\mathrm{CO}$ emission that the bulk of the gas deviates from pure Keplerian rotation (Lin et al. 2006). These perturbations and/or warps might contribute to the difficulty in firmly establishing the system's inclination. But this just raises the inevitable next question: what causes those fluctuations?

Though we find no direct evidence for any disk gap due to a protoplanet, there still remains a case for the presence of a companion somewhere around $\mathrm{AB}$ Aur: both the strong spiral 


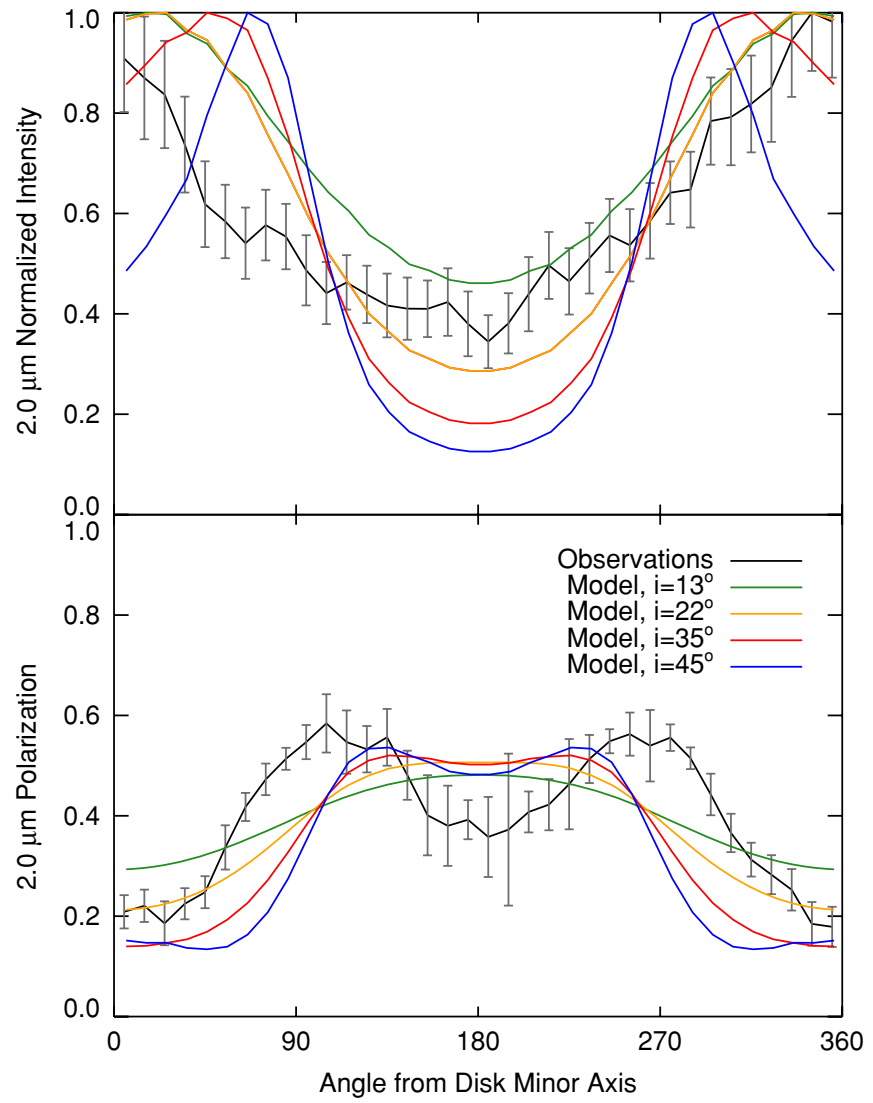

Figure 4. Observed and modeled azimuthal profiles for $2.0 \mu \mathrm{m}$ polarization and total intensity, measured in an annulus from 0.7 to $1^{\prime \prime} .5$. Azimuthal angles on the horizontal axis are measured relative to the minor axis pointing toward us. For both data and models, the near side is brightest in total intensity, but the far side is much more polarized. The models shown are for the same best-fit grain population as in Figure 3. For these dust parameters, the double-peaked pattern in polarized intensity is best fit at inclinations $\gtrsim 35^{\circ}$, but total intensity and the overall polarization level is best fit at inclinations $\sim 22^{\circ}$. We conclude that our model's $60 \%$ porous silicate spherical grains are only an approximate fit for the dust around AB Aur.

structure seen in dust-scattered light and the non-Keplerian dynamics revealed by gas emission lines argue for the existence of a planetary-mass body perturbing the disk. Other perturbation mechanisms, such as gravitational instabilities in the disk or a stellar-mass companion, seem to be ruled out observationally (Piétu et al. 2005; Lin et al. 2006). The detection of this companion thus awaits future improvements in high-contrast imaging to reveal.

\subsection{Interpreting Features in Imaging Polarimetry}

Differential polarimetry, as used by Opp08 and others, is a proven technique for observing circumstellar dust at high contrast from the ground with adaptive optics (AO). Such observations will become increasingly common with upcoming extreme AO systems, such as the Gemini Planet Imager (Macintosh et al. 2006) and SPHERE (Beuzit et al. 2006). For many disks, these instruments will provide high-contrast images only in polarized light.

We have shown here that care is required when interpreting such data. Variation of polarization with scattering angle cannot be neglected, even for low inclination targets like AB Aur. Yet AO differential polarimetry can still yield precise measurements of disk structure and dust properties, provided the degeneracy between polarization fraction and intensity can be broken. This may be possible through multiwavelength observations, which are differently sensitive to scattering geometry (Watson et al. 2007), or in combination with independent constraints on geometry such as from $\mathrm{CO}$ emission.

For brighter disks, extreme AO should allow accurate measurement of polarization fraction from the ground, but for now, HST NICMOS offers the highest polarimetric precision for measurements of disk-scattered light.

Based on observations made with the NASA/ESA Hubble Space Telescope. Support for program GO-11155 was provided by NASA through a grant from the Space Telescope Science Institute, which is operated by the Association of Universities for Research in Astronomy, Inc., under NASA contract NAS 5-26555. M.D.P. is supported by an NSF Astronomy \& Astrophysics Postdoctoral Fellowship. G.S. and D.H. were also supported by programs HST/GO 10847 and 10852. C. Pinte acknowledges the funding from the European Commission Seventh Framework Program as a Marie Curie Intra-European Fellow (PIEF-GA-2008-220891). M.D.P. thanks Ben Oppenheimer and Misato Fukagawa for discussions, and for sharing their data in FITS format.

\section{REFERENCES}

Beuzit, J.-L., et al. 2006, Messenger, 125, 29

Corder, S., et al. 2005, ApJ, 622, L133

Das, H. S., et al. 2008, MNRAS, 390, 1195

Dodson-Robinson, S. E., et al. 2009, arXiv:0909.2662

Dominik, C., et al. 2007, in Protostars and Planets V, ed. B. Reipurth, D. Jewitt, \& K. Keil (Tucson, AZ: Univ. Arizona Press), 783

Dorschner, J., et al. 1995, A\&A, 300, 503

Draine, B. T., \& Lee, H. M. 1984, ApJ, 285, 89

Eisner, J., et al. 2004, ApJ, 613, 1049

Fukagawa, M., et al. 2004, ApJ, 605, L53

Grady, C. A., et al. 1999, ApJ, 523, L151

Graham, J. R., et al. 2007, ApJ, 654, 595

Hines, D. C., et al. 2000, PASP, 112, 983

Jang-Condell, H. 2009, ApJ, 700, 820

Jang-Condell, H., \& Boss, A. 2007, ApJ, 659, L169

Kuchner, M. J., \& Holman, M. J. 2003, ApJ, 588, 1110

Kuhn, J., et al. 2001, ApJ, 553, L189

Lin, S.-Y., et al. 2006, ApJ, 645, 1297

Macintosh, B., et al. 2006, Proc. SPIE, 6272E

Mathis, J. S., \& Whiffen, G. 1989, ApJ, 341, 808

Nero, D., \& Bjorkman, J. 2009, ApJ, 702, L163

Oppenheimer, B. R., et al. 2008, ApJ, 679, 1574

Perrin, M. D., et al. 2008, PASP, 120, 555

Perrin, M. D., et al. 2009, in AIP Conf. Proc. 1158, 2009 Subaru International Conference, ed. T. Usuda, M. Tamura, \& M. Ishii (Melville, NY: AIP), 17 Petrova, E. V., et al. 2000, Icarus, 148, 526

Piétu, V., et al. 2005, A\&A, 443, 945

Pinte, C., et al. 2006, A\&A, 459, 797

Pinte, C., et al. 2008, A\&A, 489, 633

Roberge, A., et al. 2001, ApJ, 551, L97

Schneider, G., \& Hines, D. C. 2007, in the Spirit of Bernard Lyot: The Direct Detection of Planets and Circumstellar Disks in the 21st Century, ed. P. Kalas (Berkeley, CA: Univ. California), 22

Schneider, G., Silverstone, M. D., \& Hines, 2005, ApJ, 629, L117

Shen, Y., et al. 2009, ApJ, 696, 2126

Tannirkulam, A., et al. 2008, ApJ, 689, 513

Tinbergen, J. 1996, Astronomical Polarimetry (Cambridge: Cambridge Univ. Press)

Watson, A. M., et al. 2007, in Protostars and Planets V, ed. B. Reipurth,

D. Jewitt, \& K. Keil (Tucson, AZ: Univ. Arizona Press), 523

Weingartner, J. C., \& Draine, B. T. 2001, ApJ, 548, 296

Wyatt, M. C. 2005, A\&A, 440, 937 